\title{
A CURE FOR THE ENERGY SPREAD INCREASING RELATED BUNCH LENGTHENING IN ELECTRON STORAGE RINGS
}

\author{
J. Gao, LAL, B.P. 34, F-91898 Orsay cedex, France
}

\section{Abstract}

Having revealed the cause of the energy spread increasing and the corresponding bunch lengthening in electron storage rings in ref. 1, in this paper, we will propose a cure for the suppression of these collective effects. It is suggested to install an active rf cavity in the ring with the working rf wavelength about four to five times the rms bunch length. The limitation about this cure is also discussed. Finally, in the postscript, we will give an empirical bunch lengthening equation and compare the analytical results with some experimental results.

\section{INTRODUCTION}

In modern electron colliders, such as B and Tau-C Factories, short bunch lengths are required in order to get high luminosities. In practice, the bunch length will suffer from two kinds of lengthening effects. The first one is due to the potential well distortion and the second is due to the socalled collective random excitation (CRE) revealed in ref. 1. According to ref. 1 one has two equations for the energy spread increasing $\left(\mathbf{R}_{\varepsilon}=\sigma_{\varepsilon} / \sigma_{\varepsilon 0}\right)$ and the bunch lengthening $\left(\mathbf{R}_{z}=\sigma_{z} / \sigma_{z_{0}}\right)$

$$
\begin{gathered}
\mathbf{R}_{\varepsilon}^{2}=1+\frac{\mathcal{C}\left(R_{a v} R I_{b} \mathcal{K}_{\|,, 0}^{\text {tot }}\right)^{2}}{\gamma^{7} \mathbf{R}_{z}^{2.42}} \\
\mathbf{R}_{z}^{2}=1+\frac{C_{P W D} I_{b}}{\mathbf{R}_{z}^{1.5}}+\frac{\mathcal{C}\left(R_{a v} R I_{b} \mathcal{K}_{\|, 0}^{\text {tot }}\right)^{2}}{\gamma^{7} \mathbf{R}_{z}^{2.42}}
\end{gathered}
$$

where

$$
\mathcal{C}=\frac{576 \pi^{2} \epsilon_{0}}{55 \sqrt{3} \hbar c^{3}}
$$

$\epsilon_{0}$ is the permittivity in vacuum, $\hbar$ is Planck constant, $c$ is the velocity of light, $I_{b}=e N_{e} c / 2 \pi R_{a v}, R_{a v}$ is the average radius of the ring, $R$ is the bending radius, $\mathcal{K}_{\|, 0}^{\text {tot }}$ is the total longitudinal loss factor at the zero current bunch length, $C_{P W D}$ represents potential well distortion effect, $N_{e}$ is the electron number in a bunch, and $\gamma$ is the normalized particle energy.

In this paper we will investigate the possibility to suppress the CRE induced bunch energy spread increasing and the corresponding bunch lengthening.

\section{CURE: INTRODUCING AN ACTIVE RF CAVITY IN THE RING}

A bunch of $N_{e}$ particles inside will has a definite short range wake potential profile corresponding to its charge distribution. What should be stressed is that for a given bunch current even the longitudinal position of each electron inside the bunch is a random variable, the collective wake potential profile remains stable. As shown in ref. 1, after each turn an electron will get a net kick on its energy by

$$
\Delta E_{i}=\mathcal{U}_{w} \sin \left(\Omega_{s} t-\phi_{i}\right)
$$

where $\phi_{i}$ is a random variable, $\Omega_{s}$ is the angular synchrotron oscillation frequency, and $\mathcal{U}_{w}=e^{2} N_{e} \mathcal{K}_{\| !}^{\text {tot }}\left(\sigma_{z}\right)$ is the average single particle energy loss due to short range wakefield per turn. It is these random energy fluctuations which are responsible for the bunch energy spread increasing. The idea to suppress these kicks is to create an artificial counteract kicks $-e V_{0} \sin \left(\Omega_{s} t-\phi_{i}\right)$. Since it is known that an electron will get maximum positive kicks at the very head and the tail of the bunch, and the maximum negative kick near the middle of the bunch as shown in Fig. 1a, one can use a standing wave rf cavity with the wavelength of the working mode (with rf input power) about four to five times the rms bunch length. The phase relation of this mode with respect to the passing bunch should be arranged as follows: The head and the tail gets the maximum energy reduction by $-e V_{0}$ each turn and the middle of the bunch gets the maximum energy gain $e V_{0}$ per turn as shown in Fig. $1 \mathrm{~b}$ (in a practical point of view, the phase shift should be adjustable to adapt different shape of wake potentials). Obviously, to cancel the collective random kicks one has to choose $V_{0}=\mathcal{U}_{w} / e$ which is the peak voltage gain of an electron passing through the cavity. The required rf input power can be easily obtained by

$$
P_{0}=V_{0}^{2} / R_{s h}
$$

where $R_{s h}$ is the shunt impedance of the standing wave $\mathrm{rf}$ cavity. Once this is done, eqs. 1 and 2 will be changed to

$$
\begin{gathered}
\mathbf{R}_{\varepsilon}^{2} \approx 1 \\
\mathbf{R}_{z}^{2} \approx 1+\frac{C_{P W D}^{*} I_{b}}{\mathbf{R}_{z}^{1.5}}
\end{gathered}
$$

where $\left|C_{P W D}^{*}\right|>\left|C_{P W D}\right|$ due to the additional impedance contribution from the rf cavity. Till now the CRE induced energy spreading increasing and the related bunch lengthening effects have been suppressed.

As a natural rule one has to pay for what he gains, and this time it is the transverse instability.

\section{LIMITATION COMING FROM THE TRANSVERSE INSTABILITY}

Here, we will make a quick review of the single bunch transverse instability in electron storage rings. The threshold bunch current for the transverse instability derived in 
ref. 2 is expressed as:

$$
I_{b, \text { gao }}^{t h}=\frac{4 f_{y} \sigma_{\varepsilon 0} \mathbf{R}_{\varepsilon}\left|\xi_{c, y}\right|}{e<\beta_{y, c}>\mathcal{K}_{\perp}^{t o t}\left(\sigma_{z}\right)}
$$

where $\nu_{y}$ is the vertical betatron oscillation tune, respectively, $\left\langle\beta_{y, c}\right\rangle$ is the average beta function in the rf cavity region, $\xi_{c, y}$ is the chromaticity in the vertical plane (usually positive to control the head-tail instability), and $\mathcal{K}_{\perp}^{\text {tot }}\left(\sigma_{z}\right)$ is the total transverse loss factor over one turn. This threshold current indicates the disappearance of Landau damping. On the other hand, the usually used Zotter's formula coming from the mode coupling theory gives

$$
I_{b, z o t t e r}^{t h}=\frac{F f_{s} E_{0}}{e<\beta_{y, c}>\mathcal{K}_{\perp}^{t o t}\left(\sigma_{z}\right)}
$$

where $F$ is variable depending on bunch length, $f_{s}$ is the synchrotron oscillation frequency, and $E_{0}$ is the particle energy. To compare eq. 8 with eq. 9 one can write eq. 8 in a similar form as eq. 9

$$
I_{b, \text { gao }}^{t h}=\frac{F^{\prime} f_{s} E_{0}}{e<\beta_{y, c}>\mathcal{K}_{\perp}^{t o t}\left(\sigma_{z}\right)}
$$

with

$$
F^{\prime}=4 \mathbf{R}_{\varepsilon}\left|\xi_{c, y}\right| \frac{\nu_{y} \sigma_{\varepsilon 0}}{\nu_{s} E_{0}}
$$

where $\nu_{s}$ is the synchrotron oscillation tune. Apparently, if $F^{\prime}<F, I_{b, \text { gao }}^{t h}<I_{b, z o t t e r}^{t h}$ and this situation can always be realized experimentally by reducing $\left|\xi_{c, y}\right|$. If, however, eq. 9 and eq. 10 describe the same physical process, one finds the explicit expression of $F\left(F=F^{\prime}\right)$.

The impact of the longitudinal motion on that of the transverse one can be perceived by finding $\mathbf{R}_{\varepsilon}$ and $\sigma_{z}$ in eq. 8. Obviously, better longitudinal bunch properties (smaller energy spread and shorter bunch length) result in lower transverse instability threshold current.

\section{DISCUSSION}

Recent experiments on super-ACO [4] show that by using negative momentum compaction factor $\alpha$ one can get shorter bunch length, and unfortunately, larger energy spread compared with positive $\alpha$ case. This result is easily understood by looking at eqs. 1 and 2 . With negative $\alpha, C_{P W D}$ becomes negative, and in consequence, one gets smaller $\mathbf{R}_{z}$ and larger $\mathbf{R}_{\varepsilon}$. Now, if one uses the additional active $\mathrm{rf}$ cavity proposed in section 2 , eqs. 1 and 2 are reduced to eqs. 6 and 7. Obviously, $\mathbf{R}_{z}$ and $\mathbf{R}_{\varepsilon}$ are decoupled from each other, and hopefully, one can get at the same time smaller energy spread and shorter bunch length. As far as the rf cavity design is concerned, one should manage to avoid the cavity beam pipe being excessively small. The detailed discussion on the cavity design will be made after the cure principle shown in this paper be justified by the numerical simulations.

\section{CONCLUSION}

An additional active $\mathrm{rf}$ cavity is proposed to be installed in the ring to suppress the collective random excitation induced energy spread increasing and the corresponding bunch lengthening. The wavelength of the working mode should be four to five times the rms bunch length. One should be cautious to the transverse instability since smaller energy spread and shorter bunch length will result in lower transverse instability threshold current. It could be interesting to use this technique together with negative momentum compaction factor to obtain short bunch with small energy spread.

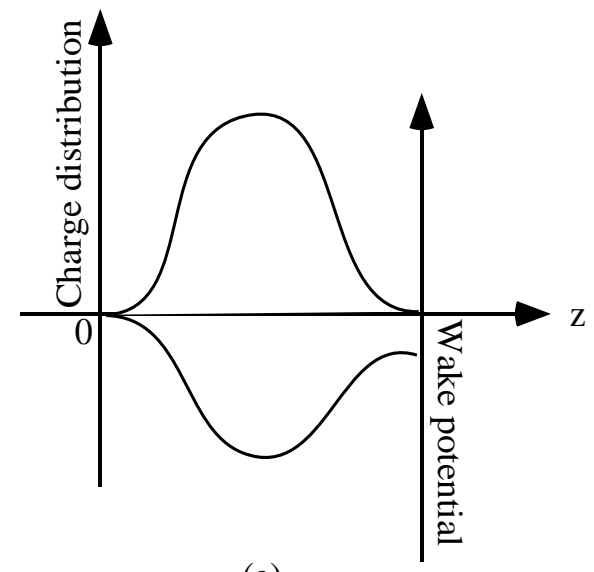

(a)

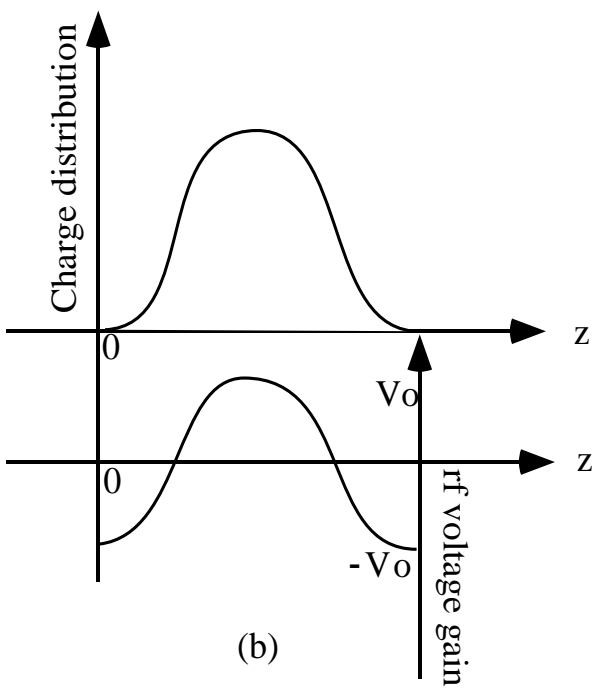

Figure 1: (a) Schematic showing of the bunch charge distribution and the short range wake potential. (b) Schematic showing of the rf voltage gain with respect to the particle position within the bunch.

\section{POSTSCRIPT}

Here, exceptionally, we add a section of "postscript" where an empirical bunch lengthening equation is given as:

$$
\mathbf{R}_{z}^{2}=1+\frac{\sqrt{2 \mathcal{C}} R_{a v} R \mathcal{K}_{\|, 0}^{\text {tot }} I_{b}}{\gamma^{3.5} \mathbf{R}_{z}^{1.21}}+\frac{\mathcal{C}\left(R_{a v} R I_{b} \mathcal{K}_{\|, 0}^{\text {tot }}\right)^{2}}{\gamma^{7} \mathbf{R}_{z}^{2.42}}
$$


An interesting property of this formula is that $\mathcal{K}_{\|, 0}^{\text {tot }}$ plays a unique dominating role. The reason for my calling this equation as empirical is because the second term on the right side of eq. 12 is not derived on a theoretical base. In Figs. 2 to 6 we make the comparison between the analytical and the experimental results of different machines. More detailed explications and comparison results can be found in ref. 5

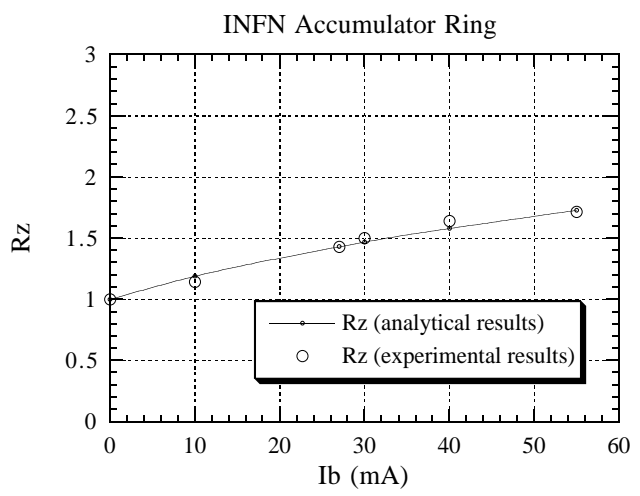

Figure 2: Comparison between INFN ( $R=1.15 \mathrm{~m}$ and $R_{a v}=5 \mathrm{~m}$ ) experimental results and the analytical results at $510 \mathrm{MeV}$ with $\sigma_{z_{0}}=3.57 \mathrm{~cm}$ and $\mathcal{K}_{\|, 0}^{\text {tot }}=0.39 \mathrm{~V} / \mathrm{pC}$.

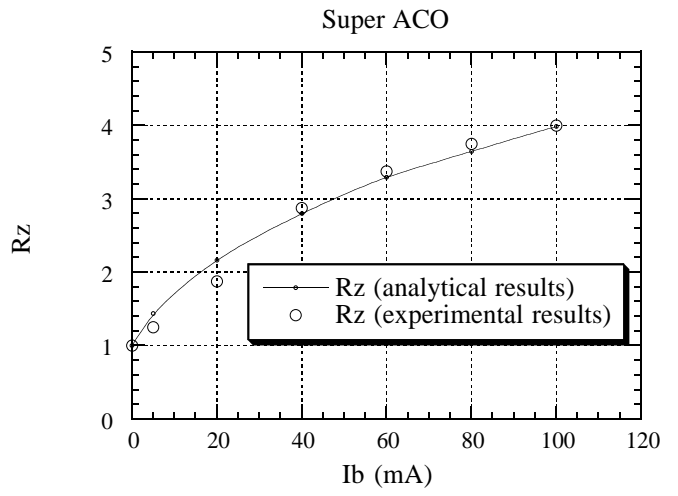

Figure 3: Comparison between Super-ACO $(R=1.7 \mathrm{~m}$ and $R_{a v}=11.5 \mathrm{~m}$ ) experimental results and the analytical results at $800 \mathrm{MeV}$ with $\sigma_{z_{0}}=2.4 \mathrm{~cm}$ and $\mathcal{K}_{\|, 0}^{\text {tot }}=3.1 \mathrm{~V} / \mathrm{pC}$.

\section{REFERENCES}

[1] J. Gao, "Bunch lengthening and energy spread increasing in electron storage rings", Nucl. Instr. and Methods, A418 (1998), p. 332.

[2] J. Gao, "Theory of single bunch transverse collective instabilities in electron storage rings", Nucl. Instr. and Methods, A416 (1998), p. 186.

[3] B. Zotter, LEP note 363 (1982).

[4] A. Nadji, P. Brunelle, G. Flynn, M.-P. Level, M. Sommer, H. Zyngier, "Experiments with low and negative momentum compaction factor with super-ACO", Proceedings of EPAC96, Barcelona, Spain, 1996.

[5] J. Gao, "An empirical equation for bunch lengthening in electron storage rings", to be published.

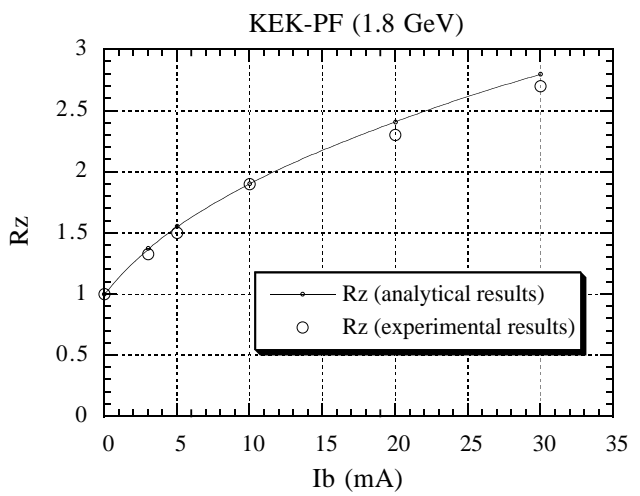

Figure 4: Comparison between KEK-PF ( $R=8.66 \mathrm{~m}$ and $R_{a v}=29.8 \mathrm{~m}$ ) experimental results and the analytical results at $1.8 \mathrm{GeV}$ with $\sigma_{z_{0}}=1.47 \mathrm{~cm}$ and $\mathcal{K}_{\|, 0}^{\text {tot }}=5.4 \mathrm{~V} / \mathrm{pC}$.

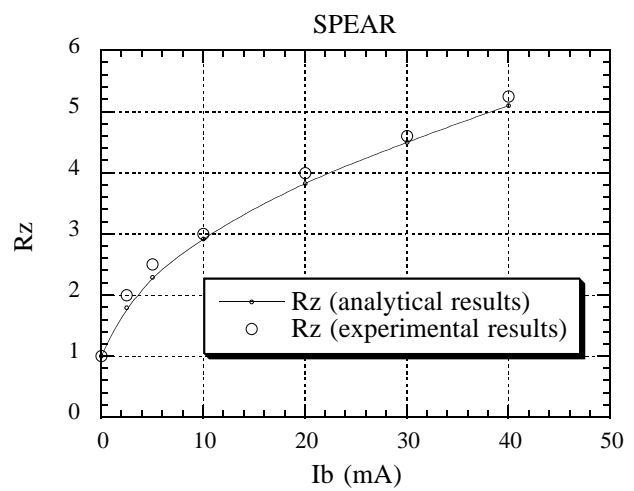

Figure 5: Comparison between SPEAR $(R=12.7 \mathrm{~m}$ and $R_{a v}=37.3 \mathrm{~m}$ ) experimental results and the analytical results at $1.5 \mathrm{GeV}$ with $\sigma_{z_{0}}=1 \mathrm{~cm}$ and $\mathcal{K}_{\|, 0}^{\text {tot }}=5.2 \mathrm{~V} / \mathrm{pC}$.

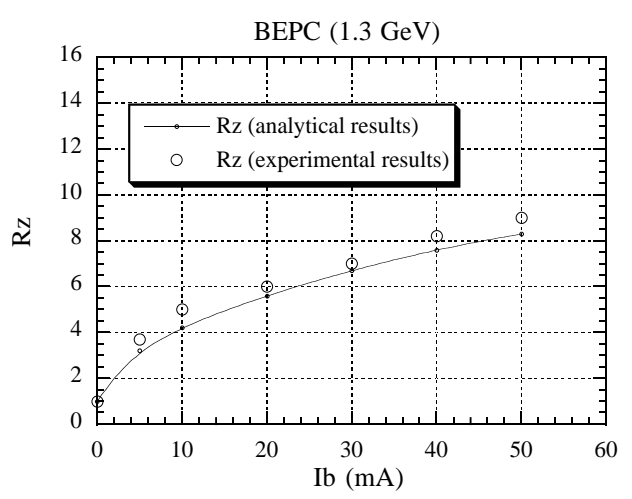

Figure 6: Comparison between BEPC (1.3 GeV) $(R=$ $10.345 \mathrm{~m}$ and $R_{a v}=38.2 \mathrm{~m}$ ) experimental results and the analytical results at $1.3 \mathrm{GeV}$ with $\sigma_{z_{0}}=1 \mathrm{~cm}$ and $\mathcal{K}_{\|, 0}^{\text {tot }}=9.6$ $\mathrm{V} / \mathrm{pC}$. 